\title{
Análise da variabilidade da frequência cardíaca durante uma sessão de fisioterapia em um paciente com insuficiência cardíaca crônica na fase I da reabilitação cardiovascular Heart rate variability during one session of physical therapy in a patient with chronic heart failure in phase $I$ of cardiac rehabilitation
}

Flávia Cristina Rossi Caruso, Ft.*, Michel Silva Reis, Ft.**, Renata Gonçalves Mendes, Ft.**, Valéria Papa, Ft. M.Sc.***, Audrey Borghi-Silva, D.Sc.****

*Especialista em Fisioterapia Cardiorrespiratória, **Doutorando em Fisioterapia pela Universidade Federal de São Carlos (UFSCar), ***Chefe do Serviço de Fisioterapia do Hospital São Francisco de Ribeirão Preto,

***Docente dos cursos de graduação e pós-graduação em Fisioterapia da Universidade Federal de São Carlos (UFSCar)

\section{Resumo}

Introdução: A função neuro-regulatória do coração tem sido investigada por meio da variabilidade da frequência cardíaca (VFC) em pacientes com insuficiência cardíaca crônica (ICC), entretanto, pouco é sabido sobre as repostas fisiológicas e da VFC nestes pacientes em ambiente hospitalar (fase I). Objetivo: Avaliar as respostas fisiológicas e da VFC durante uma sessão de fisioterapia na fase I em um paciente com ICC. Métodos: Foi avaliado um homem (54 anos), após estabilidade clínica do quadro de descompensaçáo. $\mathrm{O}$ registro da frequência cardíaca $(F C)$ foi realizado nas condiçóes supina e sentada, exercícios respiratórios (ER), deambulação e recuperaçấo. A VFC foi analisada nos domínios do tempo e da frequência. Resultados: Foi observado aumento das respostas fisiológicas e redução da VFC frente a deambulação e aumento durante os exercícios respiratórios. No entanto, na mudança postural houve aumento da atividade parassimpática. Conclusão: Uma sessão de fisioterapia pode produzir respostas fisiológicas e ajustes na modulação autonômica da FC neste paciente com ICC.

Palavras-chave: reabilitação, insuficiência cardíaca, fisioterapia.

\begin{abstract}
Introduction: The neuro-regulatory cardiac autonomic function has been investigated by heart rate variability (HRV) in patients with chronic heart failure (CHF); however, little is known about the physiologic and $\mathrm{HRV}$ responses during one session of physical therapy in phase I in CHF patients. Objective: To evaluate the physiologic and HRV responses during one session of physical therapy in phase I in CHF patient. Methods: one man (54 years) after clinical stability of $\mathrm{CHF}$ decompensation was evaluated. The heart rate records were performed in the following conditions: supine and seated position, respiratory exercises, walking and recovery. The HRV was analyzed in time and frequency domains. Results: We observed an increase of physiologic responses and a reduction of HRV during walking and an increase of HRV during respiratory exercise. However, an increase of parasympathetic activity after postural change was noticed. Conclusion: A session of physical therapy may produce physiologic responses and autonomic modulation of heart rate adjustments in this patient with CHF.
\end{abstract}

Key-words: rehabilitation, heart failure, physical therapy. 


\section{Introdução}

A função neurohumoral representa importância na fisiologia da insuficiência cardíaca crônica (ICC) [1]. A fadiga, dispneia e edema são manifestaçôes comuns, acarretando em hospitalizaçôes e alta mortalidade [2]. Neste contexto, a avaliação da frequência cardíaca (FC) pode avaliar a integridade cardíaca [3], na estratificaçáo do risco [4], na evoluçáo da doença [5], e como índice prognóstico [6] a estes pacientes.

A reabilitação cardiovascular (RCV) na fase I está indicada para otimizar o tratamento [7] e envolve mudanças posturais e exercícios que podem alterar a dinâmica cardiovascular, desencadeando em respostas compensatórias $[8,9]$. Portanto, a variabilidade da frequência cardíaca (VFC) tem sido alvo de pesquisas como método não invasivo e validado [10] em programas de RCV em ambiente ambulatorial [5].

Por outro lado, na fase hospitalar, exercícios têm sido aplicados para minimizar e reverter os efeitos deletérios do leito $[8,10]$, porém pouco é sabido sobre as respostas fisiológicas e da VFC em pacientes com ICC durante a fase hospitalar. Diante do exposto, o objetivo deste estudo foi avaliar as respostas fisiológicas e da VFC durante uma sessão de fisioterapia na fase I em um indivíduo com ICC. Nossa hipótese é de que neste paciente, uma sessão de fisioterapia pode produzir ajustes fisiológicos e da VFC.

\section{Material e método}

\section{Apresentação do caso}

Homem, 54 anos, hipertenso, sem história de tabagismo, com diagnóstico de ICC, classe funcional II. O paciente foi internado com quadro de ICC e edema agudo de pulmão, com sintomas de dispnéia e tosse com expectoração rósea. Ao exame radiológico de tórax apresentava infiltrado pulmonar à esquerda e área cardíaca aumentada. Ao ecocardiograma evidenciou-se miocardiopatia dilatada, fração de ejeção do ventrículo esquerdo de $48 \%$. Durante a internação, foi prescrito furosemida $(40 \mathrm{mg})$, digoxina $(30 \mathrm{mg})$, ramipril (30 mg). O paciente permaneceu internado por 4 dias. A avaliação ocorreu no $4^{\circ}$ dia de internação. A FC foi coletada usando um Polar S810i nas condiçóes:

1. Supina: cabeceira da cama a $30^{\circ}$ por 10 minutos;

2. Sentada: sentado por 10 minutos;

3. Exercícios respiratórios: sentado, realizou uma série de inspiraçóes e expiraçóes calmas e profundas durante 3 minutos consecutivos;

4. Deambulação: em um corredor plano por 5 minutos;

5. Recuperação: repouso sentado por 10 minutos.

Foram obtidas a pressão arterial sistólica (PAS) e diastólica, frequência respiratória, a saturaçáo periférica de oxigênio e a sensação de esforço pela escala de Borg. Os índices de VFC foram realizados no domínio do tempo (DT) pelo índice rMS-
SD (raíz quadrada da somatória do quadrado das diferenças entre os iR-R sucessivos no registro divididos pelo número de iR-R em um tempo determinado menos um), e rMSM (raíz quadrada da somatória do quadrado das diferenças dos valores individuais em relação ao valor médio dividido pelo número de iR-R em um período) [12]. No domínio da frequência (DF) foram analisados os índices de baixa frequência $(0,04-$ $0,15 \mathrm{~Hz})$ e alta frequência $(0,15-0,4 \mathrm{~Hz})$. Tais componentes foram expressos como a razáo entre eles, $\mathrm{BF} / \mathrm{AF}$ indicativa do balanço simpato-vagal.

Este estudo foi aprovado pelo Comitê de Ética da Universidade de Araraquara, sob parecer n $\mathrm{n}^{\circ} 396$ e todos os aspectos éticos foram contemplados.

\section{Resultados}

A Tabela I ilustra as variáveis fisiológicas onde observou-se aumento da PAS (1\%) durante a deambulação. Já os índices no DT e no DF, observou-se aumento do índice RMSSD e AF durante a MASR (144\% e $100 \%$, respectivamente) quando comparado a posição supina. Porém, durante a deambulação houve aumento do índice BF/AF de 26,3\%.

\section{Discussão}

O estudo mostrou que as respostas fisiológicas e a VFC apresentou comportamento esperado frente aos exercícios respiratórios e a deambulação.

Frente a mudança postural, de supino para sentado, espera-se que ocorra aumento da atividade simpática e redução da atividade parassimpática [13]. No presente estudo, observamos maior atividade simpática na posição supina no paciente estudado. Estes resultados podem ser explicados pelo aumento do retorno venoso e congestáo pulmonar que ocorre nesta posiçáo. Este fato pode ser evidenciado pelo desconforto respiratório e dispneia que tais pacientes apresentam na posição supina [14].

Outro achado foi o aumento da atividade vagal durante os exercícios respiratórios, o qual pode ser explicado pelo estímulo das incursóes profundas e lentas provocadas pela respiração [14] sobre a modulação da FC. Neste sentido, os exercícios respiratórios podem ser estímulos importantes na redução de complicações respiratórias [15] e no controle autonômico da FC [16].

Sabendo que ajustes autonômicos são estimulados com a deambulaçáo, neste estudo, houve aumento da atividade simpática, resposta esperada durante exercícios físicos [17]. Além disso, como o índice RMSM reduziu durante o exercício, sugere-se que a deambulação pode reduzir a VFC total, sendo esta resposta também esperada em indivíduos saudáveis [18]. Neste sentido, a deambulaçâo e os exercícios respiratórios parecem ser recursos úteis para promover adaptaçôes fisiológicas, uma vez que a reabilitação pós-alta podem melhorar os índices de VFC [19] nestes pacientes. 
Tabela I - Variáveis fisiológicos e indices de VFC.

\begin{tabular}{|c|c|c|c|c|c|}
\hline & Supino & Sentado & MASR & Deambulação & Recuperação \\
\hline \multicolumn{6}{|l|}{ Variáveis Fisiológicas } \\
\hline FR & 12 & 13 & 12 & 13 & 13 \\
\hline $\mathrm{SpO}_{2}$ & $98 \%$ & $98 \%$ & $98 \%$ & $98 \%$ & $98 \%$ \\
\hline PAS $(\mathrm{mmHg})$ & 130 & 130 & 130 & 140 & 130 \\
\hline PAD $(\mathrm{mmHg})$ & 90 & 90 & 90 & 90 & 90 \\
\hline Escala de Borg (0-10) & 0 & 0 & 1 & 2 & 2 \\
\hline \multicolumn{6}{|l|}{ Índices de VFC } \\
\hline $\mathrm{rMSSD}\left(\mathrm{ms}^{2}\right)$ & 22,5 & 43,0 & 55,1 & 13,1 & 34,3 \\
\hline $\mathrm{rMSM}\left(\mathrm{ms}^{2}\right)$ & 27,3 & 16,7 & 18,4 & 2,4 & 12,1 \\
\hline $\mathrm{AF}$ (un) & 0,3 & 0,6 & 0,6 & 0,7 & 0,5 \\
\hline BF (un) & 0,7 & 0,4 & 0,8 & 0,7 & 0,5 \\
\hline $\mathrm{BF} / \mathrm{AF}$ & 1,9 & 4,0 & 0,5 & 2,4 & 1,2 \\
\hline
\end{tabular}

FR: frequência respiratória; $\mathrm{SpO}_{2}$ : saturação periférica de oxigênio; PAS: pressão arterial sistólica; PAD: pressão arterial diastólica, AF: alta frequência; BF: Baixa frequência; un: unidades normalizadas.

\section{Conclusão}

Concluímos que uma sessão de fisioterapia na fase I produziu modificaçôes fisiológicas e da VFC. No entanto, estudos com uma amostra maior devem ser propostos em ensaios futuros, com o objetivo de confirmar os achados do presente estudo.

\section{Referências}

1. Frankenstein L, Zugck C, Schellberg D, Nelles M, Froehlich $\mathrm{H}$, Katus $\mathrm{H}$ et al. Prevalence and prognostic significance of adrenergic escape during chronic b-blocker therapy in chronic heart failure. Eur J Heart Fail 2009;11:178-84.

2. Martínez-Sellés M, Martínez E, Cortés M, Prieto R, Gallego L, Fernández-Avilés F Determinants of long-term survival in patients hospitalized for heart failure. J Cardiovasc Med 2009;3.

3. Longo A, Ferreira D, Correia MJ. Variabilidade da frequência cardíaca. Rev Port Cardiol 1995;14:241-62.

4. Nessler J, Nessler B, Kitliński M, Libionka A, Kubinyi A, Konduracka $\mathrm{E}$ et al. Sudden cardiac death risk factors in patients with heart failure treated with carvedilol. Kardiol Pol 2007;65:1417-22.

5. Wu GQ, Arzeno NM, Shen LL, Tang DK, Zheng DA, Zhao NQ et al. Chaotic signatures of heart rate variability and its power spectrum in health, aging and heart failure. PLoS One 2009; $4: 4323$.

6. Smilde TD, van Veldhuisen DJ, van den Berg MP. Prognostic value of heart rate variability and ventricular arrhythmias during 13-year follow-up in patients with mild to moderate heart failure. Clin Res Cardiol 2009;98:233-9.

7. Leon AS, Franklin BA, Costa F, Balady GJ, Berra KA, Stewart $\mathrm{KJ}$ et al. Cardiac rehabilitation and secondary prevention of coronary heart disease: an American Heart Association scientific statement from the Council on Clinical Cardiology and the Council on Nutrition, Physical Activity, and Metabolism, in collaboration with the American association of Cardiovascular and Pulmonary Rehabilitation. Circulation 2005;111:369-76.

8. Matsunaga A, Masuda T, Ogura MN, Saitoh M, Kasahara Y, Iwamura $\mathrm{T}$ et al. Adaptation to low-intensity exercise on a cycle ergometer by patients with acute myocardial infarction undergoing phase I cardiac rehabilitation. Circ J 2004;68:938-45.

9. Nunan D, Donovan G, Jakovljevic DG, Hodges LD, Sandercock GR, Brodie DA. Validity and reliability of short-term heart-rate variability from the Polar S810. Med Sci Sports Exerc 2009; 41:243-50.

10. Burtin C, Clerckx B, Robbeets C, Ferdinande P, Langer D, Troosters $\mathrm{T}$ et al. Early exercise in critically ill patients enhances short-term functional recovery. Crit Care Med. 2009;37:2499505.

11. Heart rate variability. Standards of measurement, physiological interpretation, and clinical use. Task Force of the European Society of Cardiology and the North American Society of Pacing and Electrophysiology. Circulation 1996;93:1043-65.

12. Carnethon MR, Liao D, Evans GW, Cascio WE, Chambless LE, Rosamond WD et al. Does the cardiac autonomic response to postural change predict incident coronary heart disease and mortality? The atherosclerosis risk in communities study. Am J Epidemiol 2002;155:48-56.

13. Löllgen D, Mueck-Weymann M, Beise RD. The deep breathing test: median-based expiration-inspiration difference is the measure of choice. Muscle Nerve 2009;39:536-44.

14. Yánez-Brage I, Pita-Fernández S, Juffé-Stein A, MartínezGonzález U, Pértega-Díaz S, Mauleón-García A. Respiratory physiotherapy and incidence of pulmonary complications in off-pump coronary artery bypass graft surgery: an observational follow-up study. BMC Pulm Med 2009;28;9:36.

15. Swanson KS, Gevirtz RN, Brown M, Spira J, Guarneri E, Stoletniy $L$. The effect of biofeedback on function in patients with heart failure. Appl Psychophysiol Biofeedback 2009;34(2):71-91.

16. Cay S. Heart rate recovery after exercise: An important prognostic criterion. Int J Cardiol 2009;14.

17. Braunschweig F, Linde C, Adamson PB, Magalski A, Erdmann E, Kjellstrom B et al. Continuous central hemodynamic measurements during the six-minute walk test and daily life in patients with chronic heart failure. Eur J Heart Fail 2009;11:594-601.

18. Piotrowicz E, Baranowski R, Piotrowska M, Zieliński T, Piotrowicz R. Variable effects of physical training of heart rate variability, heart rate recovery, and heart rate turbulence in chronic heart failure. Pacing Clin Electrophysiol 2009;32:113-5. 